\title{
Navigating the Binary: A Visual Narrative of Trans and Genderqueer Menstruation
}

\author{
S. E. Frank and Jac Dellaria
}

An understanding of trans and genderqueer perspectives on menstruation is essential to contribute to a non-pathologizing discourse about trans and genderqueer bodies and experiences. ${ }^{1}$ Menstruation is embodied and intertwined with social expectations, norms and stereotypes of femininity.

The research on which these comics are based blends online ethnography and 19 interviews with trans and genderqueer emerging adults (ages 18-29) who recounted their embodied experiences with menstruation. Interviewees lived across the United States with a heavy concentration in Wisconsin and Chicago. Online articles and blogs $(n=16)$ were analyzed along with public social media posts tagged \#transmenstruation, \#bleedingwhiletrans $(\mathrm{n}=530)$. The data yield that menstruation manipulates social interactions for trans and genderqueer people in four dominant realms of social life: (1) gender/sex identity (2) public bathroom attendance and navigation, (3) product marketing and messaging, and (4) healthcare. Each of these arenas is permeated by the biologically and socially constructed gender/sex binary, and as a result trans and genderqueer menstruators confront preexisting constraints ranging from social interactions to the built environment.

The first comic (Fig. 7.1) illustrates how menstruation can impact gender identity for trans and genderqueer people. Given the interactional development of the self and the body as a socio-historical politic, trans and genderqueer people who menstruate must contest their identity with the the persistance of the socially constructed female body in interactions with individuals and institutions. Trans and genderqueer people who menstruate face such discourse from family, friends, advertisers, product manufacturers and 


\section{ON IDENTITY}
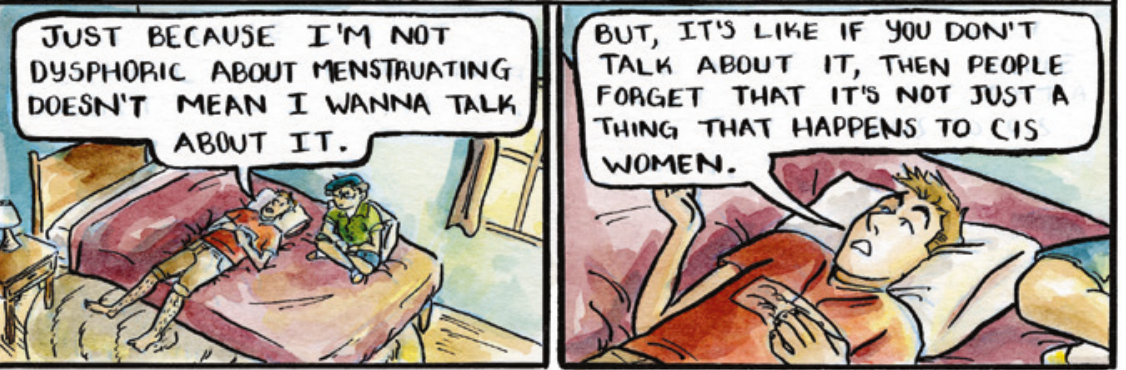

I FEEL KINDA STUCK BECAUSE

PERIODS ARE SUCH "A WOMAN'S

THING" THAT IF I SPEAK UP,

THEN I'LL BE SEEN AS

INVALIDATING MY IDENTITY.

BUT IF I DON'T, THEN NO ONE

WILL EVER LEARN.

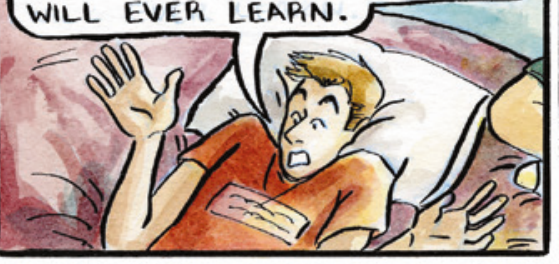

I MEAN, I DON'T REALLY

HAVE MUCH DYSPHORIA EXCEPT ON MY PERIOD.
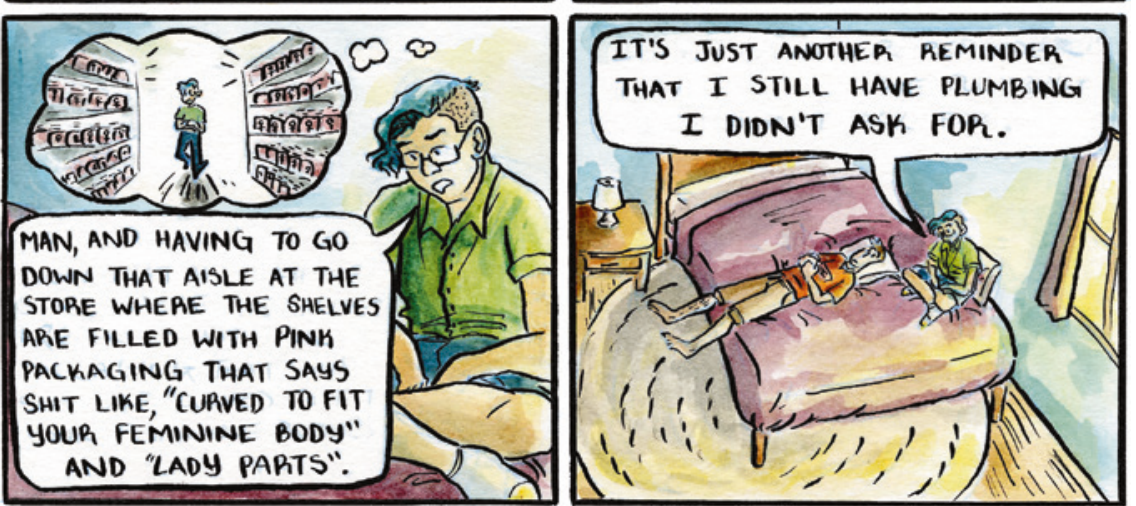

JAC DELLARIA 2019

Fig. 7.1 On Identity (Credit: Jac Dellaria. 2019) 

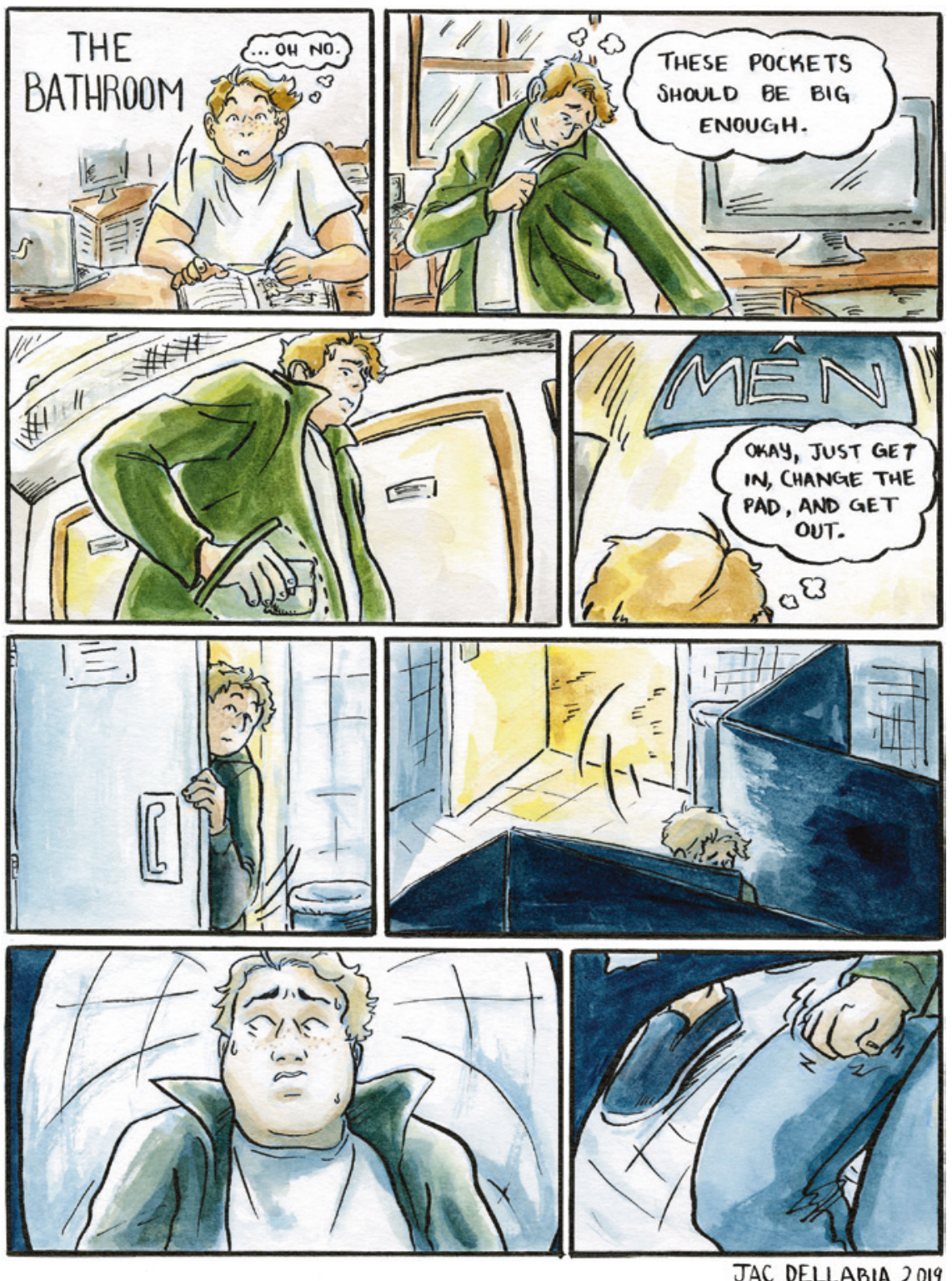

Fig. 7.2 The Bathroom (Credit: Jac Dellaria. 2019) 

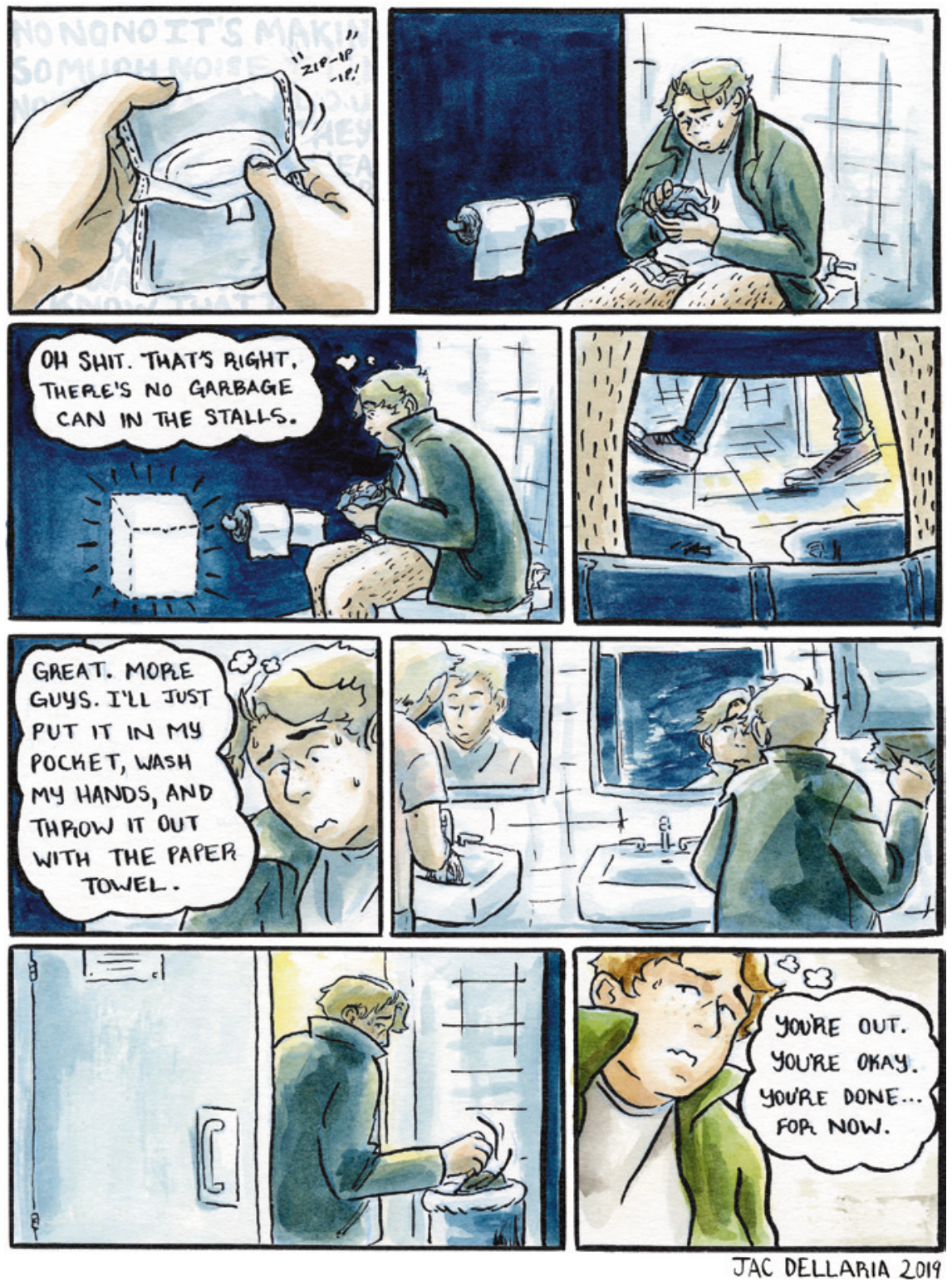

Fig. 7.3 The Bathroom (Credit: Jac Dellaria. 2019) 


\section{PRODUCT PROBLEMS}

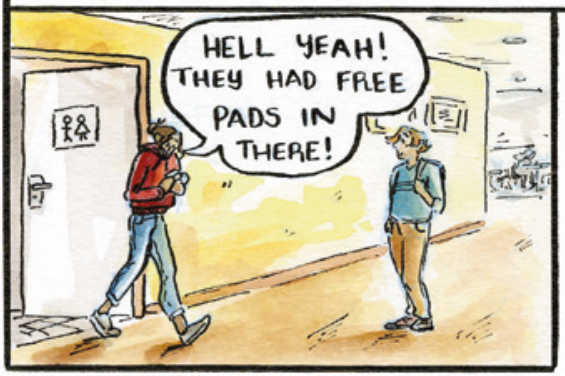

AH, I TRIED A MENSTRUAL CUP, BUT I'D RATHER NOT HAVE TO REACH IN THERE AND TOUCH ANYTHING MORE THAN I HAVE TO, SO I JUST STICK TO PADS.
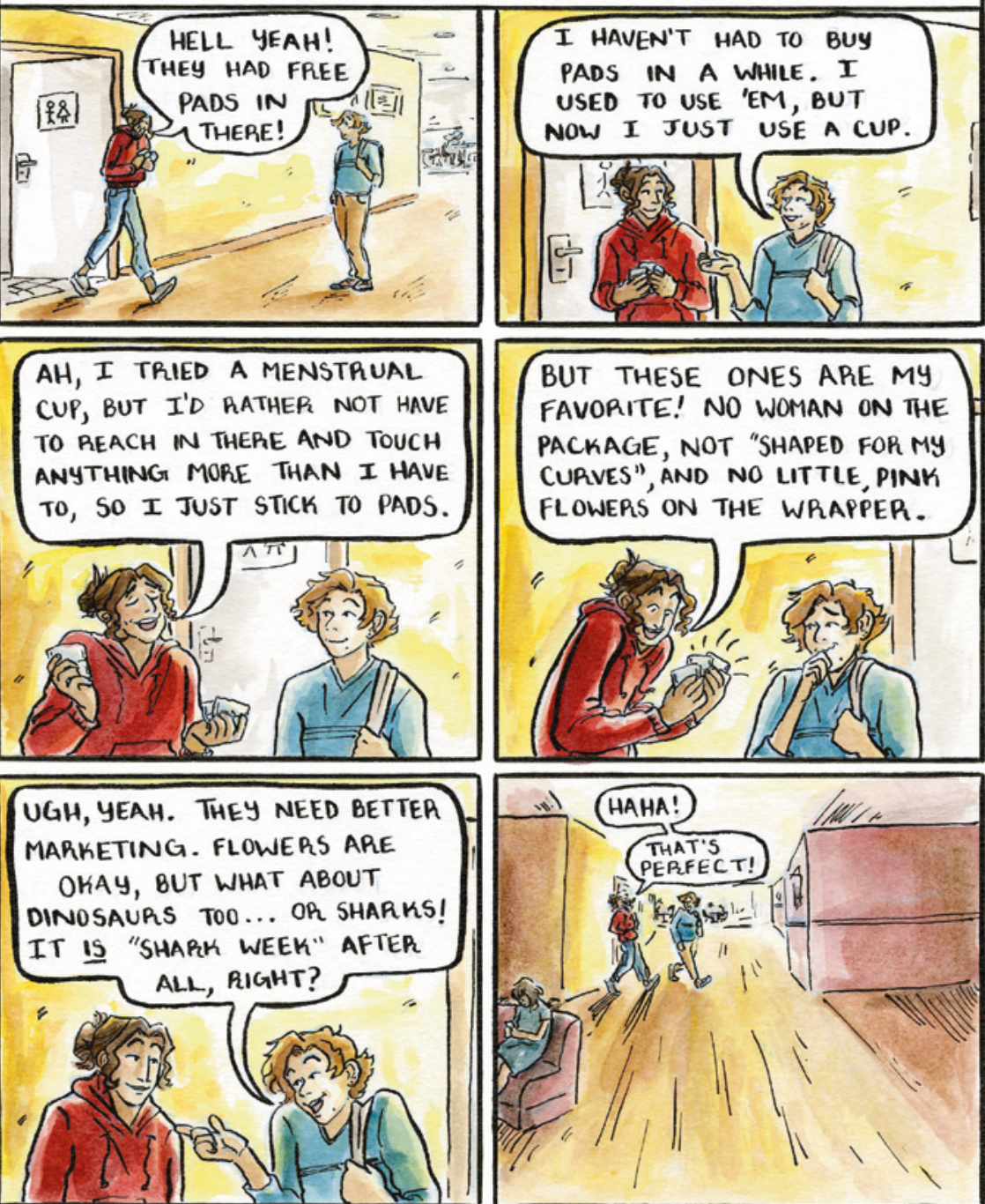

BUT THESE ONES ARE MY FAVORITE! NO WOMAN ON THE PACKAGE, NOT "SHAPED FOR MY CURVES", AND NO LITTLE, PINK FLOWERS ON THE WRAPPER.
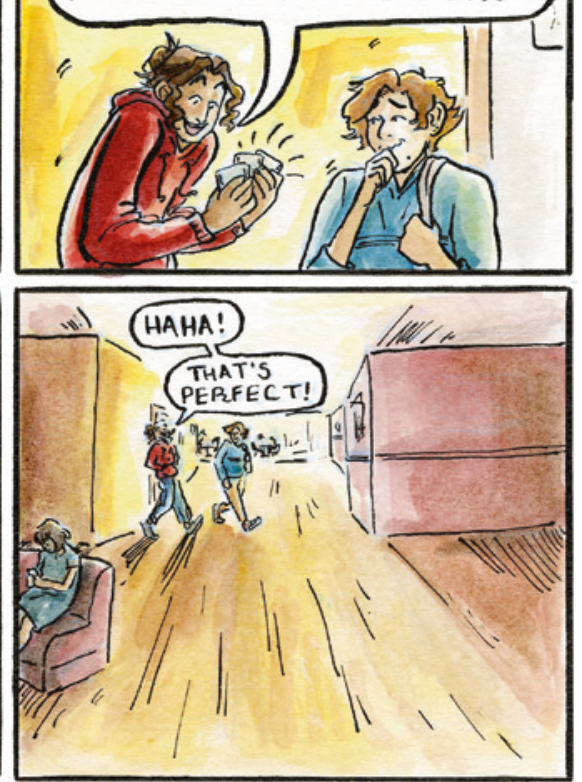

JAC DELLARIA 2019

Fig. 7.4 Product Problems (Credit: Jac Dellaria. 2019) 

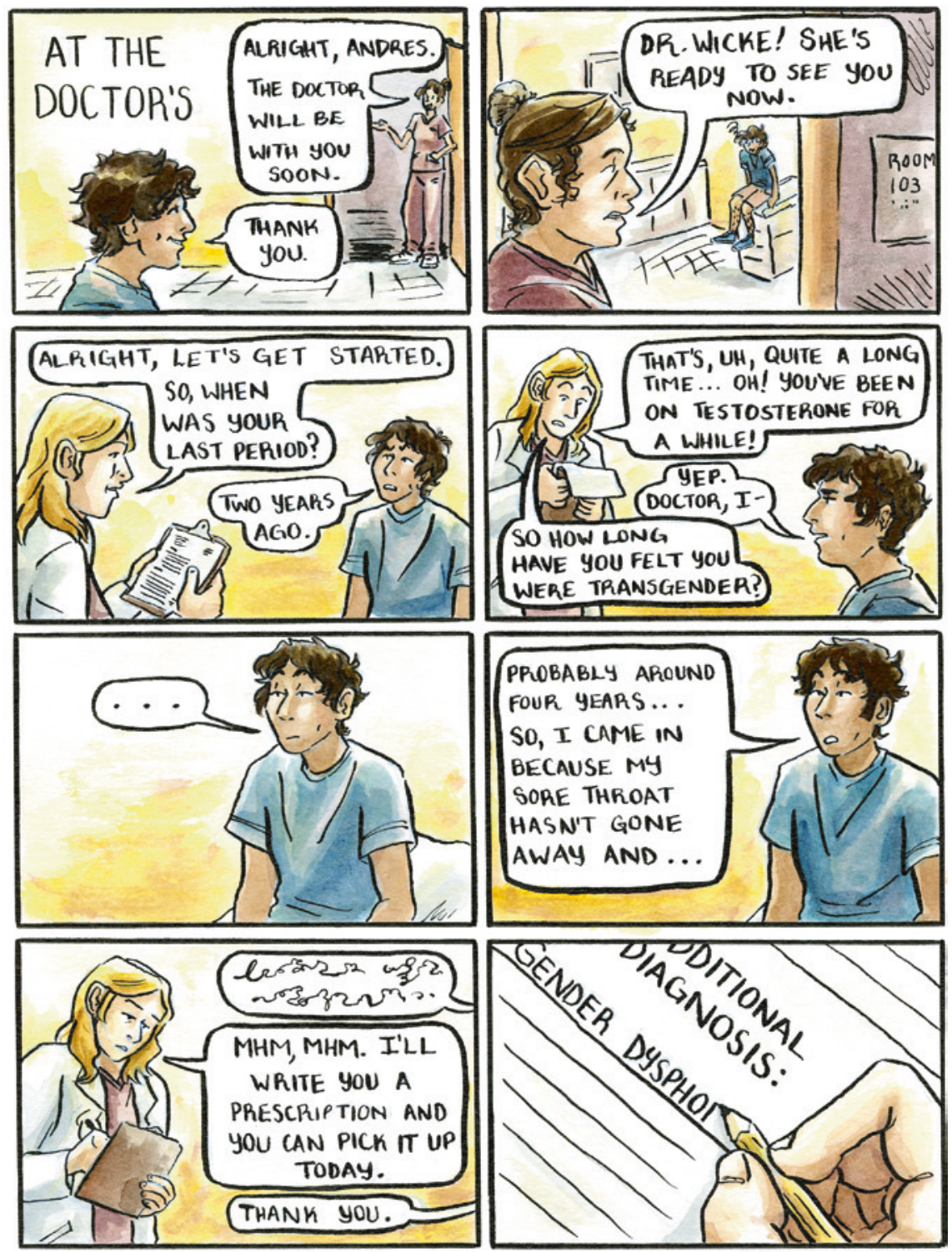

JAC DELLARIA 2019

Fig. 7.5 At the Doctor's (Credit: Jac Dellaria. 2019) 
signage throughout public spaces, which often serve as constant reminders of the contested self.

The second comic (Figs. 7.2 and 7.3) reveals bathrooms as sites of contested gender/sex identity in the context of menstruation. The rules and symbols that govern bathrooms as gender/sex policed spaces rouse fears and anxieties for many trans and genderqueer people. Menstrual products are gendered/sexed as products for women/females and so products are rarely made available in men's restrooms. Structurally, the stalls in men's rooms rarely have disposal receptacles, which signaled to participants that their bodies are unwelcome in men's spaces. In addition, the audible sound of opening a pad or tampon posits a risk that a trans/genderqueer person might reveal their status as someone who menstruates and thus does not adhere to the binary of gender and sex alignment. Trans and genderqueer persons employ coping strategies for avoiding this noise, such as opening a menstrual product before entering the bathroom, waiting to change a menstrual product until bathrooms are empty or seeking out a gender-neutral bathroom-strategies that burden menstruators with heightened awareness, vigilance, and anxiety.

The third comic (Fig. 7.4) shifts the focus to the feminization of menstrual products. Packaging, designs, commercials, and "feminine product" aisles in stores serve as regular reminders to trans and genderqueer people that a biological aspect of their bodies is deeply tied to social norms and expectations of femininity and womanhood.

The fourth comic (Fig. 7.5) illustrates generally negative interactions with healthcare providers. Even when doctors and healthcare staff attempt to include trans and genderqueer identities and bodies in their practices, efforts are often uncoordinated. Many trans and genderqueer persons experience misgendering by one or more of the medical staff or administration. Moreover, doctors and nurses ask about menstruation when it was irrelevant to the visit, which can be triggering for some people. Other people have been on testosterone for years making menstruation impossible.

Taken together, the research and comics illustrate the gendered symbolic interactions trans and genderqueer people must navigate in their daily lives. These micro social symbols of gender/sex distinction are symptoms of a larger gender regime in which gender/sex are interpreted, regulated, and policed.

\section{Note}

1. "Navigating the Binary: A Visual Narrative of Trans and Genderqueer Menstruation" S.E. Frank and Jac Dellaria was published in an extended form as Frank S. E. (2020) “Queering Menstruation: Trans and Non-Binary Identity and Body Politics." Sociological Inquiry 90 (2). Reprinted with permission. [OA CC-BY 4.0]. 
Open Access This chapter is licensed under the terms of the Creative Commons Attribution 4.0 International License (http://creativecommons.org/licenses/ by $/ 4.0 /)$, which permits use, sharing, adaptation, distribution and reproduction in any medium or format, as long as you give appropriate credit to the original author(s) and the source, provide a link to the Creative Commons license and indicate if changes were made.

The images or other third party material in this chapter are included in the chapter's Creative Commons license, unless indicated otherwise in a credit line to the material. If material is not included in the chapter's Creative Commons license and your intended use is not permitted by statutory regulation or exceeds the permitted use, you will need to obtain permission directly from the copyright holder. 